\title{
Efeito antialimentar de extratos hidroalcoólicos de Melia azedarach (Meliaceae) sobre Helicoverpa armigera (Lepidoptera: Noctuidae)
}

\author{
Jessica Velasco Andradeㅁ, Thamiris Penajo Alcassio' ${ }^{1}$, Fabíola Gonzaga Saldanha ${ }^{1}$, Luciana \\ Cláudia Toscano ${ }^{1}$, Pamella Mingotti Dias ${ }^{2}$
}

\begin{abstract}
${ }^{1}$ Universidade Estadual de Mato Grosso do Sul - UEMS, Cassilândia, Mato Grosso do Sul, Brasil. E-mail: jessikandrade2010@hotmail.com, thamirisalcassio@gmail.com, fabiolagonzag@yahoo.com.br, toscano@uems.br

${ }^{2}$ Universidade Federal de Mato Grosso do Sul - UFMS, Chapadão do Sul, Mato Grosso do Sul, Brasil. E-mail: pamellamingotti@hotmail.com
\end{abstract}

Recebido: 27/12/2015; Aceito: 29/03/2016.

\section{RESUMO}

O objetivo desse trabalho foi avaliar o efeito antialimentar de extrato hidroalcoólico de Melia azedarach em Helicoverpa armigera. $\mathrm{O}$ delineamento utilizado foi inteiramente casualizado com quatro tratamentos e cinco repetições em parcelas subdivididas. Os tratamentos utilizados foram: extratos de folhas, ramos, folhas + ramos de M. azedarach a $5 \%$ (p/v) e água destilada (testemunha), nos quais discos foliares de milho foram imersos e utilizados para a alimentação de lagartas de $H$. armigera. Foram realizados testes com e sem chance de escolha, onde avaliaram-se as variáveis: número de insetos atraídos, consumo de massa seca (g) e índice de preferência (\%). Para o teste com chance de escolha foram observadas diferenças significativas para a atratividade apenas quando utilizou-se extrato de folhas + ramos de $M$. azedarach. De acordo com a classificação de índice de preferência, os extratos de folhas $(-0,2)$ e folhas + ramos $(-0,6)$ apresentaram-se repelentes. No teste sem chance de escolha foi observado menor número de lagartas atraídas em discos foliares tratados com extrato vegetal de folhas quando comparado a testemunha. Da mesma forma o consumo de massa seca (g) de discos foliares de milho tratados com extratos vegetais de folhas e folhas + ramos foi reduzido. Conclui-se que os extratos de folhas e folhas + ramos de M. azedarach são eficientes para a repelência de H. armigera.

Palavras-chave: Planta inseticida. Antixenose. Milho. Lagartas.

\section{Effect antialimentary of extracts hydroalcoholic of Melia azedarach (Meliaceae) on Helicoverpa armigera (Lepidoptera: Noctuidae)}

\begin{abstract}
The objective of this study was to evaluate the effect antialimentary of hydroalcoholic extract of Melia azedarach on Helicoverpa armigera. An completely randomized with four treatments and five replications in a split plot was used. The treatments were: leaf extracts, branches, leaves + branches of $M$. azedarach at 5\% (p/v) and distilled water (control), in which maize leaf discs were immersed and used for feeding of $H$. armigera caterpillars. Tests were performed with and without choice, where the variables are evaluated: number of attracted insects, dry matter intake $(\mathrm{g})$ and preference index $(\%)$. According to the preference index classification, the leaf extracts $(0.2)$ and leaves + branches (-0.6) presented themselves repellents. In the no-choice test was observed fewer attracted caterpillars on leaf discs treated with plant extract of leaves when compared to control. Similarly, the dry matter intake $(\mathrm{g})$ of maize leaf discs treated with plant extracts of leaves and leaves + branches were reduced. The results indicate that the extracts of leaves and leaves + branches of $M$. azedarach are effective repellency to H. armigera.
\end{abstract}

Key words: Pesticide plant. Antixenosis. Maize. Caterpillars 


\section{Introdução}

A Helicoverpa armigera (Lepidoptera: Noctuidae) é uma praga exótica com alto poder destrutivo devido a características como, polifagia, podendo atacar várias culturas, facilidade migratória, elevada fertilidade e adaptação a diferentes ambientes. As fêmeas são capazes de ovipositar de 1000 a 1500 ovos durante todo seu ciclo de vida e até 400 ovos em apenas uma noite (ALI; CHOUDHURY, 2009).

O período larval desse noctuídeo dura em torno de 30 dias e é caracterizado pelo desenvolvimento de seis instares. A medida que crescem as lagartas adquirem colorações variadas. Em culturas agrícolas o seu ataque ocorre desde a emergência até a colheita, se alimentando de estruturas vegetativas e reprodutivas da planta (CZEPAK et al., 2013). Esse lepidóptero causou diversos prejuízos na safra 2012/2013, onde foi relatado principalmente nas culturas de soja, algodão e milho. Nessa safra a $H$. armigera causou grandes transtornos, principalmente nos estados da Bahia, Mato Grosso, Paraná e Distrito Federal (SPECHT et al., 2013).

$\mathrm{Na}$ tentativa de reduzir os danos ocasionados pela $H$. armigera, diversos inseticidas foram utilizados. Porém, o uso de forma incorreta dessas substâncias químicas pode acarretar em desequilíbrios ambientais e gerar maiores custos de produção. Com base nisso, o MIP vem buscar alternativas que minimizem os impactos causados em áreas agrícolas, como por exemplo, a utilização de inseticidas orgânicos naturais (BRUNHEROTTO; VENDRAMIM, 2001; GOMES et al., 2014).

As Meliáceas destacam-se como um importante grupo de plantas inseticidas, dentre as quais, o cinamomo Melia azedarach L., tem sido estudado como uma promissora alternativa de inseticida botânico (VENDRAMIM et al., 2000).

A aplicação de extratos botânicos para o controle de insetos-praga é uma alternativa para minimizar os impactos negativos no agroecossistema e também um método sustentável para o pequeno agricultor (MAZZONETTO; VENDRAMIM 2003).

Diversos autores constataram a importância de extratos de Meliáceas no controle de outro noctuídeo, Spodoptera. frugiperda. Haas et al. (2012) observaram $100 \%$ de mortalidade de S. frugiperda tratadas com extrato aquoso de frutos de cinamomo, quando adicionado a dieta artificial. Bogorni e Vendramim (2005) observaram 50\% de mortalidade desta lagarta com extratos de ramos de Trichilia pallida e de folhas de Trichilia pallens. Maroneze e Gallegos (2009) avaliando extratos aquosos de folhas de $M$. azedarach nas concentrações de $1 \%$ e $5 \%$ observaram $100 \%$ de mortalidade em $S$. frugiperda.

O objetivo deste trabalho foi avaliar o efeito antialimentar do extrato hidroalcoólico de M. azedarach sobre H. armigera.

\section{Material e Metódos}

O experimento foi conduzido em sala climatizada da Universidade Estadual de Mato Grosso do Sul (UEMS), Unidade Universitária de Cassilândia (UUC).

A criação massal de $H$. armigera foi mantida em sala climatizada com a temperatura de $25^{\circ} \mathrm{C}$, umidade relativa de $70 \%$ e fotoperíodo de 12 horas e alimentadas com dieta artificial modificada por Greene et al. (1976). As lagartas utilizadas foram provenientes de criação massal, em laboratório da Unesp-Jaboticabal.

Para obtenção dos extratos, as estruturas vegetais de M. azedarach, folhas e ramos foram separadas e posteriormente levadas para secagem em estufa de circulação forçada de ar $\left(40{ }^{\circ} \mathrm{C}\right.$ por $\left.48 \mathrm{~h}\right)$, em seguida foram trituradas em moinho de facas rotativas até a obtenção de um pó fino o qual foi misturado ao álcool etílico na proporção de $10 \mathrm{~g}$ de extrato em pó de cada tratamento por $100 \mathrm{~mL}$ de álcool. Em seguida, foi agitado para homogeneização da amostra durante $2 \mathrm{~h}$ em câmara agitadora e mantido em repouso por $24 \mathrm{~h}$ sob refrigeração para extração dos compostos hidrossolúveis Vendramim et al. (2000).

Após esse período, o material foi filtrado em tecido tipo voil para retirada do material sólido, e novamente submetido a agitação para volatilização do solvente, posteriormente foi filtrado e o volume completado com água destilada para $200 \mathrm{~mL}$, obtendo uma solução hidroalcoólica na concentração de $5 \%(\mathrm{p} / \mathrm{v})$ de acordo com a metodologia proposta por Vendramim et al. (2000).

As sementes de milho híbrido P3250 (PIONNER) foram semeadas em vasos plásticos de $8 \mathrm{~L}$, com solo e esterco bovino na proporção 1:1. Para realizar os experimentos foram utilizadas folhas de milho com 25 dias de emergência, estas foram cortadas em discos com auxílio de um vazador de $2,5 \mathrm{~cm}$ de diâmetro, em seguida foram imersos nas soluções hidroalcoólicas dos tratamentos de folhas, ramos, folhas+ramos e água destilada (testemunha), por 20 segundos e, após, foram distribuídos sobre papel toalha e mantidos em condição ambiente para evaporação do excesso de líquido (BRUNHEROTTO; VENDRAMIM, 2001).

Foram realizados testes com e sem chance de escolha, avaliando-se os parâmetros: número de insetos atraídos, consumo de massa seca (g) e índice de preferência (\%), calculado conforme a fórmula proposta por Procópio et al. (2003).

$$
\mathrm{IP}=(\% \mathrm{IPT}-\% \mathrm{Ipt}) /(\% \mathrm{IPT}+\% \mathrm{Ipt})
$$

onde: IP = índice de preferência; \%IPT $=\%$ de insetos no tratamento; $\% \mathrm{Ipt}=\%$ de insetos na testemunha. Valores de IP entre $-1,00$ a $-0,10$, composto teste repelente; $-0,10 \mathrm{a}+0,10$, composto teste neutro; $+0,10$ a $+1,00$, composto teste atraente. 
Para o teste de atratividade com chance de escolha, foram utilizados quatro discos foliares de milho, sendo dois tratados (extrato) e dois não tratados (água destilada), dispostos aos pares de forma cruzada e equidistantes e para o teste sem chance de escolha foram utilizados quatro discos provenientes do mesmo tratamento.

Foram utilizadas caixas tipo Gerbox $(15 \times 15 \mathrm{~cm})$ com o fundo recoberto por papel-filtro levemente umedecido com água destilada. Foi liberada 1 lagarta com aproximadamente 10 dias de idade no centro de cada Gerbox e foram realizadas avaliações após 1, 3, 6, 9, 12 e 24 horas para quantificar o número de insetos atraídos conforme metodologia de Mazzonetto et al. (2013).

Para avaliar a massa seca consumida, um disco foliar inteiro foi levado a estufa a $60^{\circ} \mathrm{C}$, durante $12 \mathrm{~h}$, para obtenção da massa seca padrão conforme a metodologia de Boiça-Júnior et al. (2013). Após 24 horas as sobras dos discos foliares consumidos pelas lagartas foram secos da mesma forma, e por meio da diferença entre a massa seca do padrão e da sobra da alimentação das lagartas chegou-se à massa seca consumida.

O delineamento experimental utilizado foi inteiramente casualizado com quatro tratamentos e cinco repetições em parcelas subdivididas, sendo as parcelas (tratamento) e subparcelas (tempo).

Os dados foram submetidos a análise de variância e as médias comparadas pelo teste " $t$ " de Student a $5 \%$ de probabilidade em teste com chance de escolha e teste Tukey a $5 \%$ de probabilidade em teste sem chance utilizado. Os dados foram transformados em $(x+0,5)^{1 / 2}$.

\section{Resultados e Discussão}

A partir de 3 horas de avaliação, observou-se que houve diferença significativa quando foram utilizados extratos vegetais de folhas + ramos de M. azedarach. Os discos foliares de milho tratados foram menos atrativos em relação à testemunha, sugerindo efeito repelente, em teste com chance de escolha (Tabela 1).

Maroneze e Gallegos (2009) avaliaram após 24 horas a atratividade de outro Lepidoptera, S. frugiperda em discos foliares de milho imersos no extrato vegetal de folhas de cinamomo nas concentrações de $0,1 \%, 1 \%$ e $5 \%$ e observaram que o extrato não exerceu efeito significativo sobre a preferência alimentar das lagartas de primeiro instar, isso também foi observado neste trabalho.

Os extratos de cinamomo testados neste trabalho não promoveram mortalidade de lagartas de $H$. armigera, o mesmo foi observado por Nunes et al. (2013) em outro Noctuidae, Chabuata major quando alimentadas com discos foliares tratados com extrato de frutos de $M$. azedarach. Entretanto, Haas et al. (2012) após 72 horas de avaliação, observaram $100 \%$ de mortalidade em lagartas de $2^{\circ}$ instar de $S$. frugiperda alimentadas com dieta artificial acrescida de $10 \%$ de extrato de frutos de M. azedarach.

Apesar do extrato vegetal de folhas e folhas + ramos de $M$. azedarach apresentarem classificação como, repelentes $(-0,2$ e -0,6) através da classificação do índice de preferência (IP), o consumo de massa seca dos discos foliares tratados com extratos vegetais de diferentes estruturas de $M$. azedarach quando foram avaliados após 24 horas, não diferiram significativamente (Tabela 2). Resultados próximos aos encontrados por Mazzoneto et al. (2013) ao utilizar extratos aquosos de folhas do Nim, Azadirachta indica (Meliaceae) também não observaram diferenças entre áreas consumidas de discos foliares de milho tratados e não tratados, mesmo que este tenha sido classificado como repelente pelo teste de IP. Vinte e quatro horas após a imersão dos discos foliares de milho nos extratos vegetais, em teste sem chance de escolha foi verificado o efeito repelente de folhas de M. azedarach para H. armigera (Tabela 3).

Tabela 1. Número médio de lagartas de H. armigera, atraídas para os discos foliares de milho tratados com diferentes extratos vegetais de M. azedarach, em teste com chance de escolha. Temp.: $25 \pm 2^{\circ} \mathrm{C}$, UR: $60 \pm 10 \%$ e Fotofase: 12 horas.

\begin{tabular}{|c|c|c|c|c|c|c|c|}
\hline \multirow{2}{*}{ Tratamentos } & \multicolumn{7}{|c|}{ Tempo (horas) } \\
\hline & 1 & 3 & 6 & 9 & 12 & 18 & 24 \\
\hline Testemunha & $0,2 \mathrm{a}$ & $0,2 \mathrm{a}$ & $0,0 \mathrm{a}$ & $0,4 \mathrm{a}$ & $0,2 \mathrm{a}$ & $0,6 \mathrm{a}$ & $0,6 \mathrm{a}$ \\
\hline \multirow[t]{2}{*}{ Folhas } & $0,0 \mathrm{a}$ & $0,4 \mathrm{a}$ & $0,2 \mathrm{a}$ & $0,0 \mathrm{a}$ & $0,2 \mathrm{a}$ & $0,4 \mathrm{a}$ & $0,4 \mathrm{a}$ \\
\hline & \multicolumn{2}{|c|}{ CV 1 (\%): 27,67} & CV 2 (\%): 26,78 & \multicolumn{2}{|c|}{ F (Trat.): $0,180^{\mathrm{ns}}$} & \multicolumn{2}{|c|}{ F (Trat. x Tempo): $0.175^{\text {ns }}$} \\
\hline Testemunha & $0,6 \mathrm{a}$ & $0,4 \mathrm{a}$ & $0,4 \mathrm{a}$ & $0,6 \mathrm{a}$ & $0,4 \mathrm{a}$ & $0,4 \mathrm{a}$ & $0,2 \mathrm{a}$ \\
\hline \multirow[t]{2}{*}{ Ramos } & $0,4 \mathrm{a}$ & $0,4 \mathrm{a}$ & $0,4 \mathrm{a}$ & $0,4 \mathrm{a}$ & $0,0 \mathrm{a}$ & $0,6 \mathrm{a}$ & $0,8 \mathrm{a}$ \\
\hline & \multicolumn{2}{|c|}{ CV 1 (\%): 31,88} & CV 2 (\%): 21,09 & \multicolumn{2}{|c|}{$\mathrm{F}$ (Trat.): $0,24^{\mathrm{ns}}$} & \multicolumn{2}{|c|}{ F (Trat. x Tempo): $0,27^{\text {ns }}$} \\
\hline Testemunha & $0,0 \mathrm{a}$ & $0,8 \mathrm{~b}$ & $0,6 \mathrm{a}$ & $0,6 \mathrm{~b}$ & $0,8 \mathrm{~b}$ & $0,8 \mathrm{~b}$ & $0,8 \mathrm{~b}$ \\
\hline \multirow[t]{2}{*}{ Folhas + Ramos } & $0,0 \mathrm{a}$ & $0,0 \mathrm{a}$ & $0,4 \mathrm{a}$ & $0,0 \mathrm{a}$ & $0,2 \mathrm{a}$ & $0,2 \mathrm{a}$ & $0,2 \mathrm{a}$ \\
\hline & \multicolumn{2}{|c|}{ CV $1(\%): 97,02$} & CV 2 (\%): 21,11 & \multicolumn{2}{|c|}{ F (Trat.): $0,23^{\text {ns }}$} & \multicolumn{2}{|c|}{ F (Trat. x Tempo): $21,11^{* *}$} \\
\hline
\end{tabular}

Médias seguidas pela mesma letra nas colunas, não diferem significativamente pelo teste " $\mathrm{t}$ " de Student a 5\% de probabilidade. ${ }^{\text {ns }}$ (não significativo); (significativo a $5 \%$ ). 
Tabela 2. Massa seca (g) de discos foliares de milho, tratados e não tratados com $M$. azedarach consumida por lagartas de $H$. armigera, em teste com chance de escolha. Temp.: $25 \pm 2^{\circ} \mathrm{C}$, UR: $60 \pm 10 \%$ e Fotofase: 12 horas.

\begin{tabular}{|c|c|c|c|c|}
\hline \multirow[b]{2}{*}{$\begin{array}{c}\text { Extratos Vegetais } \\
5 \%(\mathrm{p} / \mathrm{v})\end{array}$} & \multicolumn{2}{|c|}{ Consumo de massa seca (g) } & \multirow{2}{*}{$\begin{array}{c}\text { Índice de } \\
\text { Preferência }^{2}\end{array}$} & \multirow[b]{2}{*}{ Classificação ${ }^{2}$} \\
\hline & $\begin{array}{l}\text { Discos foliares } \\
\text { Tratados }\end{array}$ & $\begin{array}{l}\text { Discos foliares não } \\
\text { tratados }^{1}\end{array}$ & & \\
\hline Folhas & $0,005 \mathrm{a}$ & $0,007 \mathrm{a}$ & $-0,2$ & Repelente \\
\hline Ramos & $0,012 \mathrm{a}$ & $0,012 \mathrm{a}$ & 0,6 & Atraente \\
\hline Folhas + Ramos & $0,005 \mathrm{a}$ & $0,010 \mathrm{a}$ & $-0,6$ & Repelente \\
\hline F $1: 0,4^{\mathrm{ns}}$ & F $2: 0,8^{\mathrm{ns}}$ & F 3: $0,1^{\text {ns }}$ & & \\
\hline CV 1 (\%): 61,24 & CV 2 (\%): 29,08 & CV 3 (\%): 57,93 & & \\
\hline
\end{tabular}

1 Médias seguidas pela mesma letra, nas linhas, não diferem significativamente pelo teste "t" de Student a $5 \%$ de probabilidade. ns (não significativo); ** (significativo a 5\%)

2 IP $=$ Índice de preferência; Valores de IP entre $-1,00$ a - 0,10 , composto teste repelente; $-0,10$ a $+0,10$, composto teste neutro; $+0,10$ a $+1,00$ composto teste atraente.

Tabela 3. Número médio de lagartas de H. armigera, atraídas para os discos foliares de milho tratados com diferentes extratos vegetais de M. azedarach, em teste sem chance de escolha. Temp.: 25 2oC, UR: $60 \pm 10 \%$ e Fotofase: 12 horas.

\begin{tabular}{lccccccc}
\hline \multirow{2}{*}{ Tratamentos } & \multicolumn{7}{c}{ Tempo (horas) } \\
\cline { 2 - 8 } & 1 & 3 & 6 & 9 & 12 & 18 & 24 \\
\hline Testemunha & $0,40 \mathrm{a}$ & $0,60 \mathrm{a}$ & $0,60 \mathrm{a}$ & $0,80 \mathrm{a}$ & $0,80 \mathrm{a}$ & $0,80 \mathrm{a}$ & $1,00 \mathrm{~b}$ \\
Folhas & $0,00 \mathrm{a}$ & $0,20 \mathrm{a}$ & $0,20 \mathrm{a}$ & $0,80 \mathrm{a}$ & $0,40 \mathrm{a}$ & $0,20 \mathrm{a}$ & $0,20 \mathrm{a}$ \\
Ramos & $0,60 \mathrm{a}$ & $0,40 \mathrm{a}$ & $0,80 \mathrm{a}$ & $0,80 \mathrm{a}$ & $0,80 \mathrm{a}$ & $0,80 \mathrm{a}$ & $1,00 \mathrm{~b}$ \\
Folhas + Ramos & $0,20 \mathrm{a}$ & $0,20 \mathrm{a}$ & $0,60 \mathrm{a}$ & $0,60 \mathrm{a}$ & $0,80 \mathrm{a}$ & $0,60 \mathrm{a}$ & $0,60 \mathrm{ab}$ \\
\hline F (Tratamento) & $0,06^{\text {ns }}$ & & & & & & \\
CV 1 (\%) & 69,24 & & & & & \\
CV 2 (\%) & 21,36 &
\end{tabular}

Nota-se que o consumo de massa seca (g) de discos foliares de milho em teste sem chance de escolha, com extratos vegetais de folhas e folhas + ramos de M. azedarach, foram menores quando comparados à testemunha (Tabela 4). Tal fato, possivelmente se deve à compostos químicos denominados de taninos que estão presentes em maiores quantidades nas folhas de cinamomo. Segundo Monteiro et al. (2005), numa mesma espécie vegetal, a quantificação de taninos pode variar consideravelmente, devido à questão de sazonalidade, local de origem da planta e parte da planta utilizada.

Tabela 4. Massa seca (g) de discos foliares de milho tratados com M. azedarach, consumida por lagartas de H. armigera, em teste sem chance de escolha. Temp.: $25 \pm 2{ }^{\circ} \mathrm{C}$, UR: $60 \pm$ $10 \%$ e Fotofase: 12 horas.

\begin{tabular}{|c|c|}
\hline $\begin{array}{c}\text { Extratos Vegetais } \\
5 \%(\mathrm{p} / \mathrm{v})\end{array}$ & Consumo de massa seca $(\mathrm{g})$ \\
\hline Testemunha & $0,010 \mathrm{~b}$ \\
\hline Folhas & $0,005 \mathrm{a}$ \\
\hline Ramos & $0,008 \mathrm{ab}$ \\
\hline Folhas + Ramos & $0,006 \mathrm{a}$ \\
\hline $\begin{array}{l}\mathrm{F} \text { (Tratamento) } 0,003^{* *} \\
\mathrm{CV}(\%) 32,96\end{array}$ & \\
\hline
\end{tabular}

\section{Conclusões}

O extrato de folhas e folhas + ramos de $M$. azedarach em discos foliares de milho, apresentaram efeito antialimentar para $H$. Armigera.

Apesar desses extratos apresentarem efeitos promissores sobre o comportamento de $H$. armigera, novos estudos ainda devem ser realizados.

\section{Referências}

ALI, A.; CHOUDHURY, R. A. Some biological characteristics of Helicoverpa chickpea. Tunisian Journal of Plant Protection, Sousse-Tunísia, v. 4, n. 1, p. 99-106, 2009.

BOGORNI, P. C.; VENDRAMIM, J. D. Efeito subletal de extratos aquosos de Trichilia spp. sobre o desenvolvimento de Spodoptera frugiperda (J.E. Smith) (Lepidoptera: Noctuidae) em milho. Neotropical Entomology, Londrina-PR, v. 34, n. 2, p. 311-317, 2005 .

BOIÇA JÚNIOR, A. L.; JANINI, J. C.; DE SOUZA, B. H. S.; RODRIGUES, N. E. L. efeito de cultivares de repolho e doses de extrato aquoso de nim na alimentação e biologia de Plutella xylostella (Linnaeus) (Lepidoptera: Plutellidae). Bioscience Journal, Uberlândia-MG, v. 29, n. 1, p. 22-31, 2013.

BRUNHEROTTO, R.; VENDRAMIM, J. D. Bioatividade de extratos aquosos de Melia azedarach L. sobre o desenvolvimento de Tuta absoluta (Meyrick) (Lepidoptera: 
Glechiidae) em tomateiro. Neotropical Entomology, Londrina-PR, v. 30, n. 3, p. 455-459, 2001.

CZEPAK, C.; ALBERNAZ, K. C.; VIVAN, L. M.; GUIMARÃES, H. O.; CARVALHAIS, T. Primeiro registro de ocorrência de Helicoverpa armigera (Hübner) (Lepidoptera: Noctuidae) no Brasil. Pesquisa Agropecuária Tropical, Goiânia-GO, v. 43, n. 1, p. 110-113, 2013.

GOMES, A. M. A.; MARIANO, R. L. R.; SILVEIRA, E. B.; MESQUITA, J. C. P. Isolamento, seleção de bactérias e efeito da utilização de Bacillus spp. na produção de mudas orgânicas de alface. Horticultura Brasileira, Brasília-DF, v. 21, n. 4, p. 699-703, 2003.

GREENE, G. L.; LEPPLA, N. C.; DICKERSON, W. A. Velvetbean caterpillar: a rearing procedure and artificial medium. Journal of Economic Entomology, Annapolis-MDUSA, v. 69, p. 487-488, 1976.

HAAS, J.; FONTANELA, A.; HAIDA, K, S.; ALVES, L. F. A. Avaliação de extratos vegetais sobre Spodoptera frugiperda (J. E. SMITH, 1797) (Lepidoptera: Noctuidae). Revista em Agronegócios e Meio Ambiente, Maringá-PR, v. 5, n. 2, p. 353-362, 2012.

MARONEZE, D. M.; GALLEGOS, D. M. N. Efeito de extrato aquoso de Melia azedarach no desenvolvimento das fases imatura e reprodutiva de Spodoptera frugiperda (J. E. Smith, 1797) (Lepidoptera: Noctuidae). Ciências Agrárias, Londrina-PR, v. 30, n. 3, p. 537-550, 2009.

MAZZONETTO, F.; VENDRAMIM, J. D. Efeito de pós de origem vegetal sobre Acanthoscelides obtectus (Say) (Coleoptera: Bruchidae) em feijão armazenado. Neotropical Entomology, Piracicaba-SP, v. 32, n.1, p. 145-149, 2003.
MAZZONETTO, F.; CORADINI, F.; CORBANI, R. Z.; DALRI, A. B. Ação de inseticidas botânicos sobre a preferência alimentar e sobre posturas de Spodoptera frugiperda (J. E. Smith) (Lepidoptera: Noctuidae) em milho. EntomoBrasilis, Vassouras-RJ, v. 6, n. 1, p. 34-38, 2013.

MONTEIRO, J. M.; ALBUQUERQUE, U. P.; ARAÚJO, E. L. Taninos: uma abordagem da química à ecologia. Química Nova, São Paulo-SP, v. 28, n. 5, p. 892-896, 2005.

NUNES, M, Z.; SANTOS, R. S. S.; I BOFF, M. I. C.; ROSA, J. M. Bioatividade de extratos vegetais e inseticidas microbianos sobre lagartas de Chabuata major (Gueneé, 1852) (Lepidoptera: Noctuidae). Entomotropica, AraguaVEN, v. 28, n. 1, p. 11-16, 2013.

PROCÓPIO, S. O.; VENDRAMIM, J. D.; RIBEIRO JÚNIOR, J. I.; SANTOS, J. B. Bioatividade de diversos pós de origem vegetal em relação a Sitophilus zeamais Mots. (Coleoptera: Curculionidae). Ciência e Agrotecnologia, Lavras-MG, v. 27, n. 6, p. 1231-1236, 2003.

SPECHT, A.; SOSA-GOMEZ, D. R.; PAULA-MORAES, S. V.; YANO, S. A. C. Identificação morfológica e molecular de Helicoverpa armigera (Lepidoptera: Noctuidae) e ampliação de seu registro de ocorrência no Brasil. Pesquisa Agropecuária Brasileira, Brasília-DF, v. 48, n. 6, p. 689-692, 2013.

VENDRAMIM, J.D.; CASTIGLIONI, E. Aleloquímicos, resistência de plantas e plantas inseticidas. In: GUEDES, J.C. et al. (Org.). Bases e técnicas do manejo de insetos. Santa Maria-RS: Pallotti, 2000. p.113-128. 